\title{
ATAC-seq normalization method can significantly affect differential accessibility analysis and interpretation
}

\author{
Jake J. Reske ${ }^{1}$, Mike R. Wilson ${ }^{1}$ and Ronald L. Chandler ${ }^{1,2^{*}}$ (D)
}

\begin{abstract}
Background: Chromatin dysregulation is associated with developmental disorders and cancer. Numerous methods for measuring genome-wide chromatin accessibility have been developed in the genomic era to interrogate the function of chromatin regulators. A recent technique which has gained widespread use due to speed and low input requirements with native chromatin is the Assay for Transposase-Accessible Chromatin, or ATAC-seq. Biologists have since used this method to compare chromatin accessibility between two cellular conditions. However, approaches for calculating differential accessibility can yield conflicting results, and little emphasis is placed on choice of normalization method during differential ATAC-seq analysis, especially when global chromatin alterations might be expected.

Results: Using an in vivo ATAC-seq data set generated in our recent report, we observed differences in chromatin accessibility patterns depending on the data normalization method used to calculate differential accessibility. This observation was further verified on published ATAC-seq data from yeast. We propose a generalized workflow for differential accessibility analysis using ATAC-seq data. We further show this workflow identifies sites of differential chromatin accessibility that correlate with gene expression and is sensitive to differential analysis using negative controls.

Conclusions: We argue that researchers should systematically compare multiple normalization methods before continuing with differential accessibility analysis. ATAC-seq users should be aware of the interpretations of potential bias within experimental data and the assumptions of the normalization method implemented.
\end{abstract}

Keywords: ATAC-seq, Chromatin accessibility, Bioinformatics, Normalization, Genomics, Differential accessibility

\section{Introduction}

Genome-wide quantitative sequencing methods for measuring genomic features have been recently developed to address various biological questions previously limited to locus-level interrogation. Current applications include gene expression [1], DNA methylation [2], protein-DNA interactions [3], histone post-translational modifications [4], 3D genome organization [5],

\footnotetext{
*Correspondence: rlc@msu.edu

1 Department of Obstetrics, Gynecology and Reproductive Biology,

College of Human Medicine, Michigan State University, Grand Rapids, MI 49503, USA

Full list of author information is available at the end of the article
}

nucleosome occupancy [6] and chromatin accessibility [7]. Researchers frequently apply these techniques to multiple cellular states in parallel to provide biological insight into the questions being investigated. These approaches remove experimental predispositions by permitting genome-wide analysis paired with robust statistical testing procedures to improve null hypothesis rejection. As a result, there is an obvious need to benchmark and improve statistical or analytical methods used for properly interpreting the sequencing data generated by the molecular biology.

The latest major technique for effectively measuring genome-wide chromatin accessibility is the Assay for Transposase-Accessible Chromatin, or ATAC-seq. This

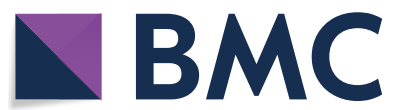

(c) The Author(s) 2020. This article is licensed under a Creative Commons Attribution 4.0 International License, which permits use, sharing, adaptation, distribution and reproduction in any medium or format, as long as you give appropriate credit to the original author(s) and the source, provide a link to the Creative Commons licence, and indicate if changes were made. The images or other third party material in this article are included in the article's Creative Commons licence, unless indicated otherwise in a credit line to the material. If material is not included in the article's Creative Commons licence and your intended use is not permitted by statutory regulation or exceeds the permitted use, you will need to obtain permission directly from the copyright holder. To view a copy of this licence, visit http://creativeco mmons.org/licenses/by/4.0/. The Creative Commons Public Domain Dedication waiver (http://creativecommons.org/publicdomain/ zero/1.0/) applies to the data made available in this article, unless otherwise stated in a credit line to the data. 
assay makes use of a $\operatorname{Tn} 5$ transposase reaction which preferentially inserts a 9-bp adapter fragment into accessible regions of the genome [8]. Adapter-ligated genomic regions, which are typically nucleosome-depleted and euchromatic, can then be enriched for sequencing. This technique provides a similar readout as DNase I hypersensitivity (DNase-seq) and formaldehyde-assisted isolation of regulatory elements (FAIRE-seq), which also measure accessible chromatin regions, and it is an orthogonal assay to Micrococcal nuclease digestion (MNase-seq), which measures nucleosome-occupied regions $[9,10]$. However, ATAC-seq offers many benefits over comparable assays including a lower input material requirement, shorter assay time, in situ library preparation, and further protocol adaptation to fresh-frozen tissue [11]. These advantages have permitted precise in vivo regulatory genomic assays on small populations of sorted cells [12-17].

ATAC-seq has been used to both identify basal accessible chromatin regions in a given cellular context as well as determine regions differentially accessible (DA) between two cellular states [18-20]. The former of which is analyzed bioinformatically through a linear process which often involves calling signal peaks throughout the genome. In this respect, the analysis framework for ATAC-seq is similar to ChIP-seq and DNase-seq [21], though few comprehensive analyses and best practice reports exist. Previous work has evaluated performance of computational methods in DNase-seq footprinting analysis, and similar efforts should be made for ATACseq [22]. For DA analysis, ATAC signal at enriched regions is quantified and compared between multiple conditions. Determining DA regions with high confidence poses a greater challenge due to variability in transposition reaction efficiency, upon which may be further compounded by in vivo heterogeneity and lack of guiding literature. The result is that outputs from multiple tools for calculating DA regions are often conflicting. Furthermore, while there are indeed a small number of studies which have attempted to streamline ATAC-seq data processing [23-26], there is little emphasis on statistical considerations of differential analysis.

Using our recently reported in vivo sorted mouse ATAC-seq data set, we show that different tools and normalization methods for calculating significant DA regions yield distinct results following disruption of a chromatin remodeler [17]. We show that qualitative techniques like MA plots (as applied in microarray analysis) can be used to identify and address global accessibility patterns which may or may not be technical in nature. We assess the DA outputs from 8 different analytical approaches and observe vastly different numbers of significant genomewide DA regions, promoter DA regions, and global accessibility trends depending on the approach used. By cross-comparing DA method outputs, we are able to define genes commonly identified as having a DA promoter across multiple approaches. Integrating RNA-seq data allowed us to determine biological relevance of each method by assessing overlap between promoter DA and differential gene expression. Next, we analyze an ATACseq data set by Schep et al. [18] and show that choice of DA method can alter biological interpretation. We also implemented negative control DA analysis in these data to highlight the sensitivity of our analysis in distinguishing signal from noise. We then propose a generalized ATAC-seq data processing workflow intended for DA analysis and supply a detailed step-by-step guide which includes example code and scripts for users. This framework includes key steps for robust comparison which reduce upstream biases, such as differing molecular complexities between libraries. We further test the ability of this workflow to identify biologically relevant peaks on a historic data set reported by original authors of this method, Buenrostro et al. [8], and show it is effective in these data. Finally, we propose a differential accessibility $\mathrm{R}$ workflow through csaw which permits testing of multiple normalization methods. In whole, researchers should be aware of differing biological interpretations resulting from different normalization methods and any biases, which may not be considered or eliminated during analysis. This is especially true wherein widespread chromatin structure alterations might be expected, such as when disrupting chromatin regulators. We further provide computational methodology that serves as a "one size fits all" guideline for ATAC-seq data analysis from reads to differential accessibility analysis.

\section{Results}

\section{Comparison of 8 analytical approaches to calculate} ATAC-seq differential accessibility

To determine if choice of ATAC-seq DA analysis method influences experimental results, we compared 8 different DA approaches (Table 1) using the published tools MACS2, DiffBind, csaw, voom, limma, edgeR, and DESeq2 [27-33]. Analyses $I$ and II follow the DiffBind protocol, originally intended for ChIP-seq data, which constructs a consensus read count matrix from MACS2 replicate peak sets of $m$ query regions by $n$ samples. Briefly, MACS2 constructs an ATAC fragment pileup from aligned paired-end data, then builds a local bias track through a series of parameters to estimate background noise, and finally compares ATAC signal to the local background at each genomic bp using a Poisson test. Significant nearby regions are then merged into a peak. DiffBind then calculates linear scaling factors from either the total number of reads in each library, which assumes 
Table 1 Description of 8 approaches used to calculate ATAC-seq differential accessibility

\begin{tabular}{|c|c|c|c|c|}
\hline \# & $\begin{array}{l}\text { Genomic } \\
\text { regions }\end{array}$ & Tool & Normalization & DA testing \\
\hline 1 & MACS2 [27] & DiffBind [29] & Full library size & DESeq2 [31] \\
\hline$\|$ & MACS2 [27] & DiffBind [29] & Reads in peaks & DESeq2 [31] \\
\hline III & MACS2 [27] & csaw [28] & TMM [34] & edgeR [30] \\
\hline IV & MACS2 [27] & csaw [28] & Loess & edgeR [30] \\
\hline V & csaw [28] & csaw [28] & TMM [34] & edgeR [30] \\
\hline VI & csaw [28] & csaw [28] & Loess & edgeR [30] \\
\hline VII & $\operatorname{MACS} 2$ [27] & $\begin{array}{l}\text { csaw [28] | voom } \\
{[32]}\end{array}$ & $\log _{2} \mathrm{Cpm}$ & limma [33] \\
\hline VIII & MACS2 [27] & $\begin{array}{l}\text { csaw [28] | voom } \\
{[32]}\end{array}$ & Quantile [35] & limma [33] \\
\hline
\end{tabular}

that true global differences may be expected and technical bias is small, or the total number of reads in queried peak regions, which should eliminate global differences in favor of reducing any technical biases. The former method is applied in $I$ and the latter in $I I$. The count matrix with normalization factors is then subject to the $D E S e q 2$ framework of dispersion estimation and negative binomial generalized linear model (GLM) fitting for hypothesis testing, according to the design matrix. Analyses $I I I$ through $V I$ follow approaches described in the csaw manual [28]. III and $I V$ count reads in query regions defined by $M A C S 2$ peak sets then filter low-abundance windows, while $V$ and $V I$ use the csaw sliding window approach to quantify ATAC signal in the 300-bp interval query windows across the genome. The de novo query windows in $V$ and $V I$ then pass low-abundance filtering and are tested for signal enrichment greater than threefold over the surrounding 2 kilobase local neighborhood. For normalization, $I I I$ and $V$ implement the trimmed mean of $M$ values (TMM [34]) method to generate linear scaling factors from counts in large, 10-kb genomic bins. This method trims the top and bottom quantiles of bins based on fold-change and signal abundance in order to minimize the changes between samples at the majority of bins. TMM assumes that most regions are not truly DA, and it assesses for systematic signal differences present across the genome that are presumed to be technical. Therefore, the TMM method should control for technical error more than scaling to total read depth by eliminating any systematic biases in library ATAC distribution, while still permitting true asymmetric differences specifically in DA regions. $I V$ and $V I$ implement a non-linear loessbased (loess: locally estimated scatterplot smoothing) normalization method. This highly conservative method normalizes the signal distribution locally based on extent of ATAC signal abundance. As a result, the loess fit assumes a symmetric global distribution in which there are no true biological global differences in ATAC reaction efficiency or distribution, and any evidence of these biases are technical and should be removed. The count matrices with respective normalization factors or offsets from all four of these csaw analyses are then subject to the edgeR statistical framework of estimating dispersions by empirical Bayes and quasi-likelihood GLM fitting for hypothesis testing, according to the design matrix. Finally, analyses VII and VIII follow the same procedure as $I I I$ and $I V$ with respect to using MACS2 peak query regions filtered by abundance in csaw, but instead they follow a voom transformation to $\log _{2}$ counts per million $\left(\log _{2} \mathrm{CPM}\right)$ in $V I I$ which are further quantile normalized in VIII. The $\log _{2}$ CPM transformation simply scales by full library size and maintains those assumptions, while quantile normalization equalizes the signal distribution across all libraries [35]. Quantile normalization should function analogously to loess normalization by eliminating any global or trended biases, and it has been previously applied to ATAC-seq data [20]. The (normalized) log-count matrices from these two analyses are then mean-variance estimated to generate weights for limma linear modeling and hypothesis testing by empirical Bayes-moderated statistics.

\section{Choice of ATAC-seq analytical approach is a key step in determining differential chromatin accessibility}

We recently reported an ATAC-seq data set in which chromatin accessibility was compared between sorted mutant and control mouse endometrial epithelial cells following disruption of a common tumor suppressor and oncogene [17]. In this in vivo study, a chromatin remodeler protein, ARID1A, was disrupted along with induced expression of a constitutively active oncoprotein, PIK3CA ${ }^{\mathrm{H} 1047 \mathrm{R}}$, resulting in myometrial [17] and peritoneal [36] invasion in $\mathrm{LtfCre}^{O /+} ;(\mathrm{Gt}) \mathrm{R}^{26^{\text {Pik3ca"H1047R; }}}$ Arid $1 a^{f l / *}$ mutant mice. Mutant and wild-type endometrial epithelial cells were positively selected by a surface marker, EPCAM, and purified by magnetic bead separation [17]. ATAC-seq was selected as a suitable method for analyzing chromatin accessibility changes in sorted cells due to feasibility of supplying low input material.

We next compared chromatin accessibility patterns between mutant and control endometrial epithelial populations using the $8 \mathrm{DA}$ approaches. Different patterns of DA measurements were observed through MA plot visualization depending on the choice of DA approach used (Fig. 1). MA plots are a type of Bland-Altman plot applied to genomic data, where they were originally used in microarray analysis [37]. MA plots depict global patterns of measurements compared between two samples, where each tested genomic feature is quantified by 


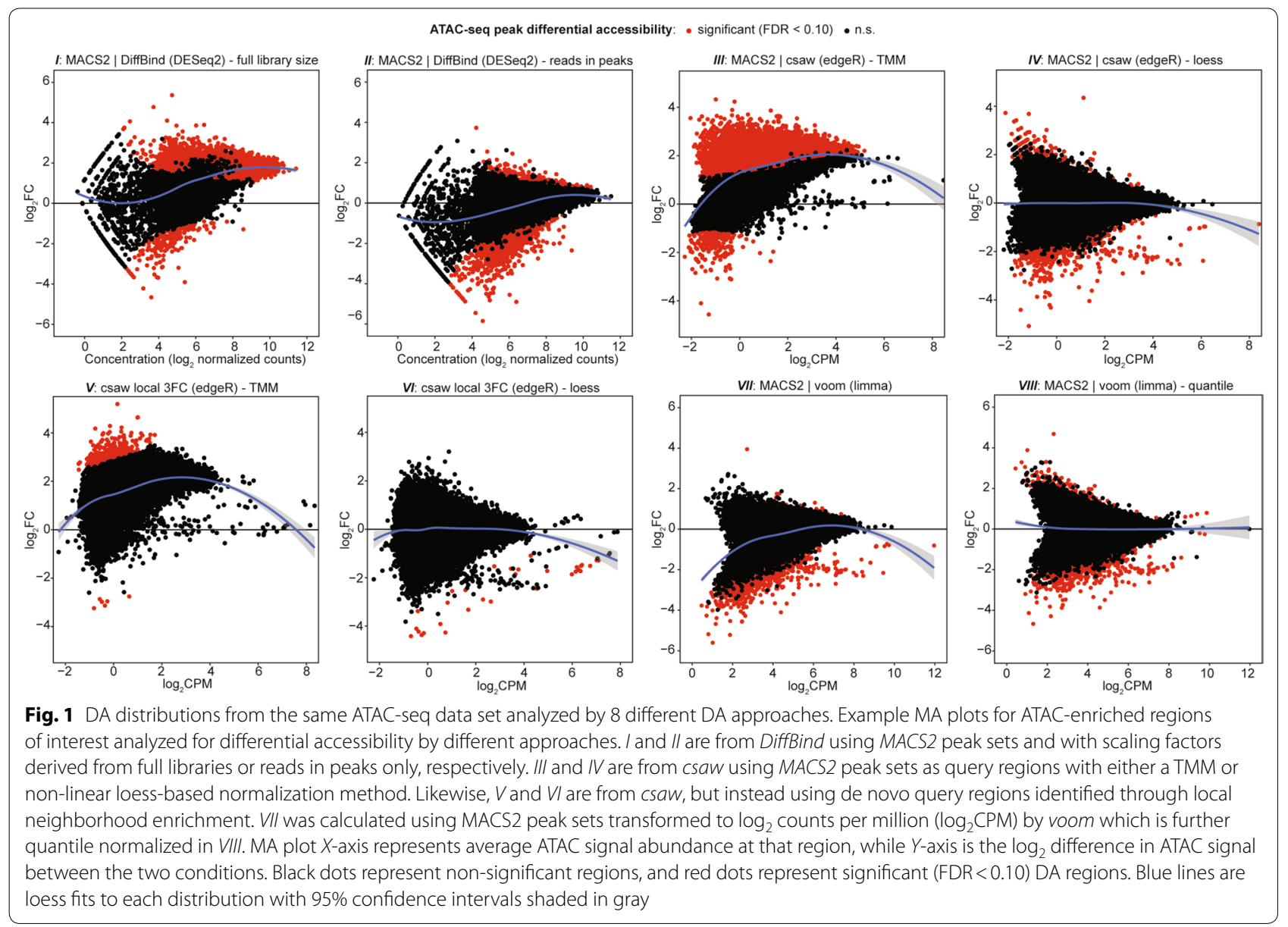

the difference between the two groups as the $y$-axis $(M)$ and signal intensity as the $x$-axis $(A)$. For example, TMM normalization of the MACS2 peaks read count matrix in III shows the majority of these genomic regions are increasing accessibility in mutant cells compared to control cells, whereas a small minority are decreasing. The TMM approach is similar to the default normalization method of DiffBind in I which scales counts based on library size, but TMM only considers regions expected to be unchanged for generating normalization factors. These observations are in contrast with the DA results of a more conservative loess-based count adjustment in $I V$. The loess normalization yielded more significant DA regions that were decreasing rather than increasing accessibility. MA plots for DA regions following all varying analyses show how these global patterns are affected through different normalization methods. The DA distribution is shifted upwards in the TMM normalized windows, which may or may not be technical in nature, and is corrected with the loess normalization.

We next performed a detailed comparative analysis of the DA outputs from all 8 different approaches.
The number of significant DA regions identified by an FDR $<0.10$ threshold ranged from 33 to 24,450 with varying proportions of regions increasing vs. decreasing accessibility (Fig. 2a). Genomic annotation of these peaks by HOMER [38] showed that gene promoters, defined as a region within $3 \mathrm{~kb}$ of a TSS, constituted varying extents of the DA regions ranging from $6 \%$ to $51 \%$ (Fig. 2b). However, in 6 out of the 8 tested approaches, gene promoters were predominantly increasing in accessibility overall (Fig. 2c). This comparative analysis permitted the probable conclusion that gene promoters are mostly increasing in accessibility in mutant cells, even though the global patterns observed in comparisons between each DA method show discordance.

Understanding what gene promoters display affected accessibility is biologically informative since promoter chromatin accessibility often correlates with transcription $[7,39]$. We first asked how many genes were commonly identified as exhibiting a DA promoter region among the multiple tested approaches. Strikingly, no genes were found commonly among all 8 approaches, though certain sets of genes were commonly identified 


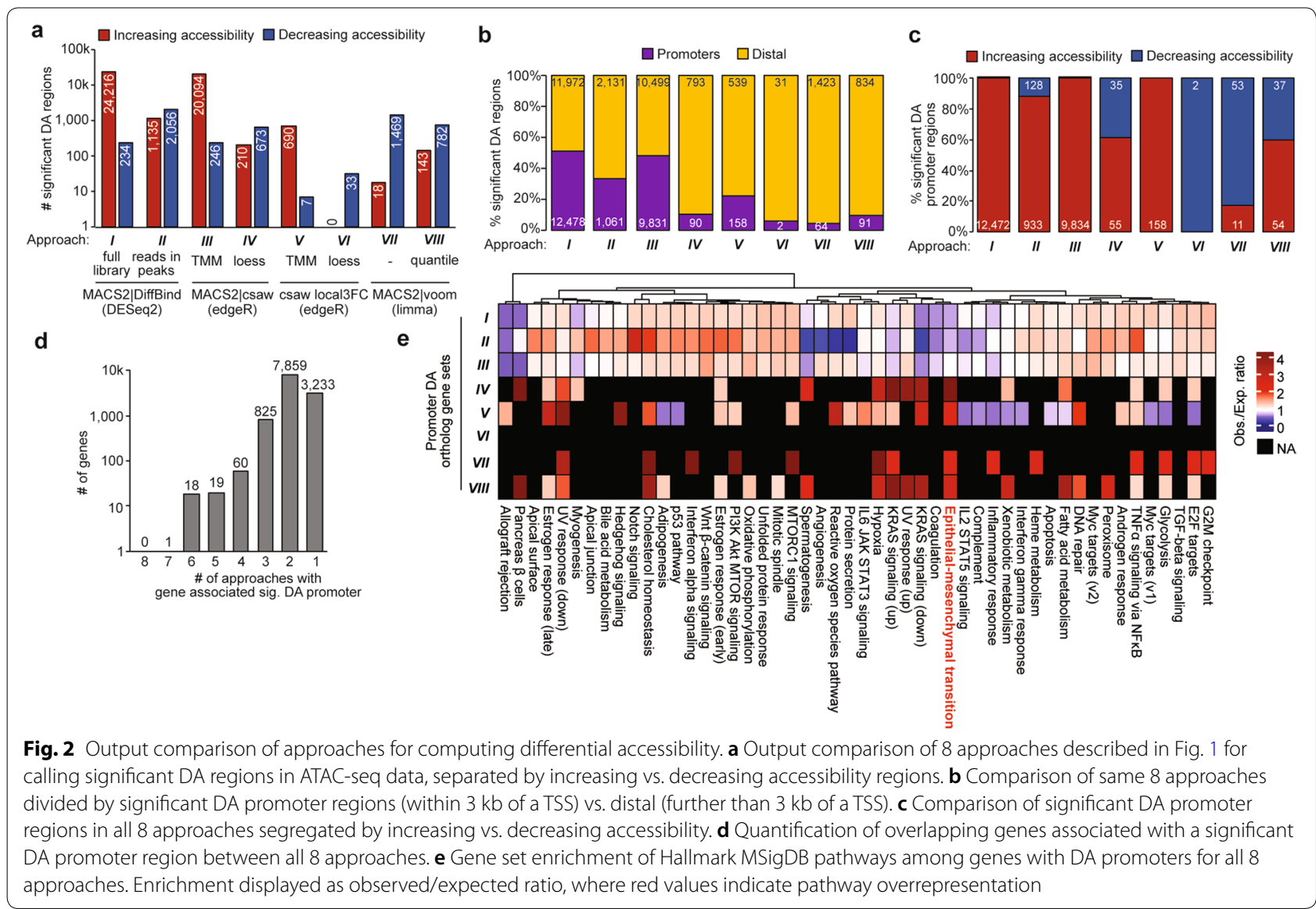

in multiple approaches (Fig. 2d). As shown by pathway enrichment analysis, choice of DA approach also led to differences in biological processes observed for promoters with affected chromatin accessibility. Epithelial-mesenchymal transition (EMT) was uniquely highlighted in significant DA promoter genes from DA approaches $I V$, $V, V I I$, and VIII as opposed to other methods (Fig. 2e). We previously reported a role of ARID1A and PIK3CA mutations in EMT-related processes through multiple molecular and cell-based in vitro and in vivo assays [17].

A commonly used approach to validate chromatin accessibility observations is to compare the results with gene expression data. Next, we asked if those genes with a significant DA promoter region found in any one of the DA approaches were associated with differential expression (DE) in mutant vs. control endometrial epithelial populations. One issue is that quantifying direct overlap between promoter DA and gene DE is sensitive to the DA FDR threshold. To overcome this, we implemented a precisionrecall (PR) curve to predict gene DE status (binary, based on RNA-seq FDR $<0.05$ threshold) with promoter DA FDR values yielded by a given DA analysis. The PR curves showed modest predictive ability of promoter DA, though analyses $I I I, I V, V$, and $V I I I$ were better predictors of gene expression changes than the others (Additional file 1: Figure S1a). The predictive abilities are most apparent before $20 \%$ recall (i.e., $20 \%$ of all DE genes with a tested promoter ATAC region), indicating that gene expression changes observed in this experiment are not entirely determined by alterations in promoter chromatin accessibility.

We further investigated how FDR thresholding could affect DA outputs. We observed different FDR thresholds between our 8 different approaches that elicited a 5\% null hypothesis rejection, ranging from $\mathrm{FDR}=0.00331$ $(I)$ to $\mathrm{FDR}=0.936(V I)$ (Additional file 1: Figure S1b). As such, we compared the number of DE genes with a DA promoter region (Additional file 1: Figure S1c) and saw that this was highly dependent on FDR threshold. These results suggest that FDR thresholding can change between DA testing methods, and optimizing this aspect of the analysis may also improve results and interpretation. Collectively, all of these analyses underscore the importance of comparative analysis with multiple DA outputs before settling on conclusions. Furthermore, 
choosing a conservative normalization method may reduce both the need for such rigorous comparisons or use of multiple independent assays.

\section{Temporal chromatin accessibility measurements in yeast also display normalization bias}

In addition to comparing the effects of genetic mutations or other treatment conditions, examining temporal changes in chromatin accessibility in cell populations is another application of ATAC-seq DA analysis. We utilized the Schep et al. [18] osmotic stress time-course ATAC-seq data set from yeast to determine if choice of DA analysis workflow yielded different results. Yeast cells were treated with $0.6 \mathrm{M} \mathrm{NaCl}$ and harvested cells for ATAC-seq at four, 15-min intervals, up to $60 \mathrm{~min}$, for comparison against control cells at 0 min exposure $(n=2$ per time point). An advantage to this data set is the inclusion of two control groups, one containing $\mathrm{NaCl}$ in the wash buffer and one without $\mathrm{NaCl}$, which permits comparisons between two negative control groups. Schep et al. also utilized a published expression microarray data set by $\mathrm{Ni}$ et al. [40] from the same $0.6 \mathrm{M} \mathrm{NaCl}$ treatment design, in which three patterns (unchanged, upregulated, and downregulated) were defined based on osmotic stress response over time. Schep et al. reported that a subset of genes from each expression response pattern also displayed similar profiles with respect to promoter accessibility change [18].

Our workflow detected between 1271 and 1894 genome-wide naïve overlap peaks at any given time condition, resulting in between 2261 and 2601 tested DA regions depending on the DA analysis approach used. DA comparison of the two control groups with all 8 analytical approaches resulted in very few statistically significant DA regions $(F D R<0.05)$, ranging from 0 to 85 regions (Additional file 1: Figure $\mathrm{S} 2$ ). This in contrast with the 15 - $\min (n=2)$ vs. 0 -min control $(n=4)$ comparison, in which between 491 and 1082 regions were determined significantly DA (Additional file 1: Figure S3a). Because the yeast genome is highly compact with functional genes and relatively few introns or other distal regulatory elements (compared to vertebrates), >95\% of DA regions at 15 min exposure were located within gene promoters (defined as -2000 to +200 bp from TSS) with every approach (Additional file 1: Figure S3b). We then determined the overlap of promoter regions displaying increasing or decreasing accessibility with each of the three gene expression patterns defined by $\mathrm{Ni}$ et al. We were able to classify DA regions and gene expression patterns for all 8 approaches, with up to $75 \%$ of the increasing accessibility promoter regions and up to $70 \%$ of the decreasing accessibility regions classified by expression changes (Additional file 1: Figure S3c). However, we noted a wide range in the number of genes displaying concordant gene expression and promoter chromatin accessibility changes (Additional file 1: Figure S3d).

The most compelling observations occurred when we assessed the full spectrum of gene expression and chromatin accessibility changes across the entire time-course series. Certain DA analyses showed biologically expected changes in overall accessibility that reflected expression, such as $I I, I V$, and VIII, while the accessibility profiles from other approaches appeared asymmetrical indicating technical bias (Fig. 3). With some DA analyses, strong DA statistical significance is observed among stably expressed genes, which are not expected to display accessibility changes (Additional file 1: Figure S4). In most cases, the MA plot profiles were predictive of the gene expression and chromatin accessibility patterns observed throughout the time course. In 4 DA methods with an asymmetrical MA plot trend (I, III, $V$, and $V I I)$, the displayed chromatin accessibility profiles over the time course did not match the direction of gene expression change (Fig. 3). Most importantly, even in a highly controlled time-course experiment in yeast, certain DA analyses can yield technically discordant results that do not align with the orthogonal assays.

\section{Generalized ATAC-seq workflow for differential chromatin accessibility analysis}

Various studies have described ATAC-seq quality control and data processing for non-computational scientists, but few emphasize DA analysis [23-26]. Moreover, we noted a literature gap in the importance of standardizing molecular complexity before quantifying differences between experimental ATAC libraries. This, coupled with our observation that choice of normalization method impacts DA results, led us to develop a comprehensive and easy-to-follow computational workflow for differential ATAC-seq data analysis.

The workflow presented in Fig. 4 is devised to be widely applicable to any ATAC-seq data set or experimental design. It is based after the standardized ENCODE pipeline devised by Kundaje et al. (https://libraries.io/githu b/kundajelab/atac_dnase_pipelines) with modifications. Example applications include calling baseline accessible regions in naïve cells and identifying DA genomic regions. The field typically accepts data sets with at minimum two biological replicates for standard peak calling, as was established by ENCODE for ChIP-seq data [41], and at least two biological replicates are absolutely required for any DA statistical analysis. For each step, we have included a descriptive phrase along with the software tools used and example code. A detailed description of the workflow is available in Additional file 1: Methods, along with a machine-readable text version with comments 


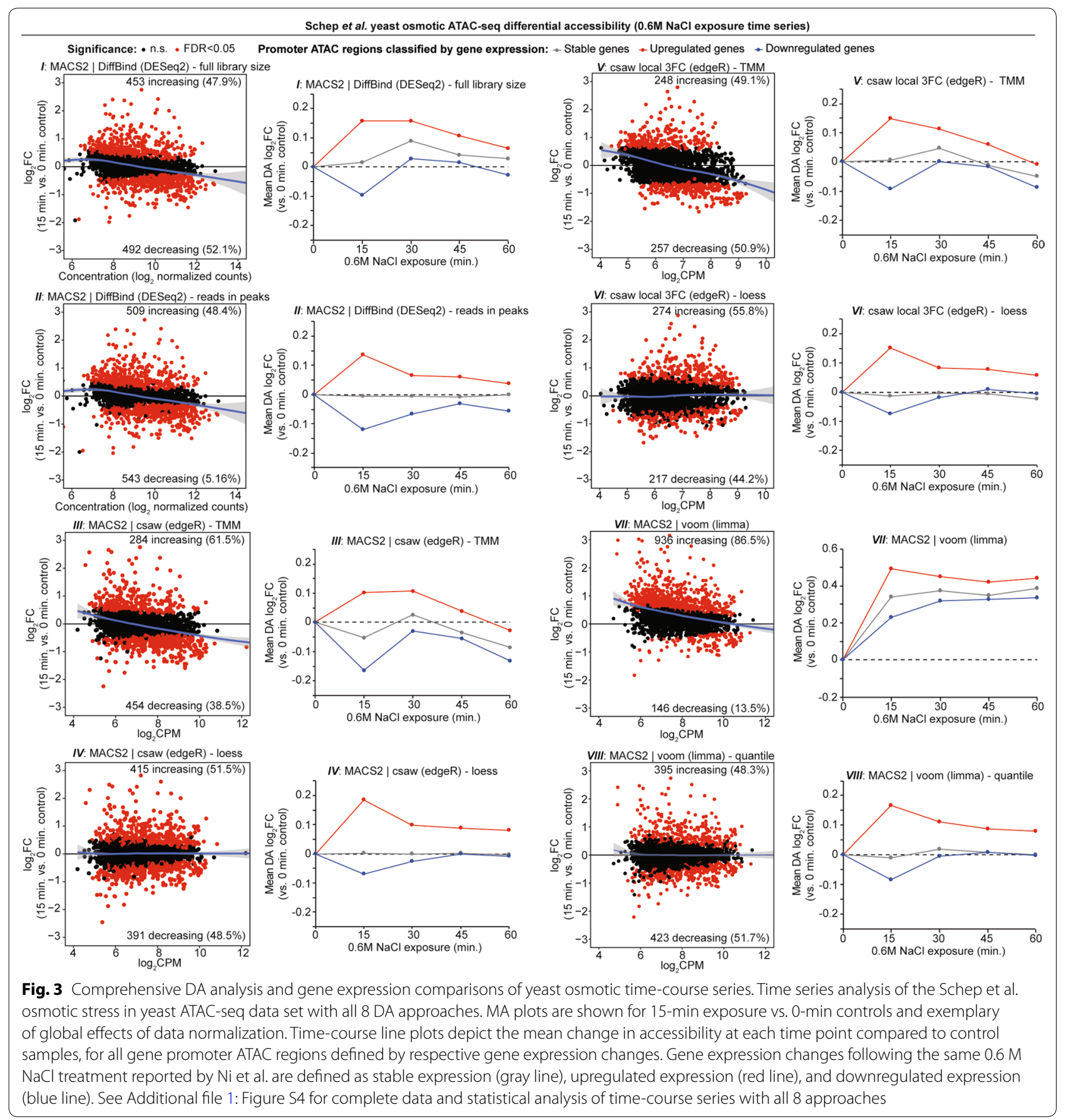

(Additional file 2). Custom Unix scripts for certain workflow functions are also supplied (Additional files 3, 4, 5). Notably, we implemented the ENCODE-defined naive overlap to determine biological replicate peak concordance. This method calls peaks on pooled replicates, and then identifies peaks displaying at least $50 \%$ overlap with all single replicate peaks. We have supplied a Unix shell script (naiveOverlapBroad.sh, Additional file 5) to execute this function for computing naïve overlap from two broadPeak replicates and can be easily modified to support more replicates. Additionally, publicly available blacklist regions (ENCODE consortium) refer to highly repetitive or unstructured regions that display artificially high signal in genomic experiments [42].

A major addition to our workflow is quantifying and normalizing library molecular complexity. Often times, it 


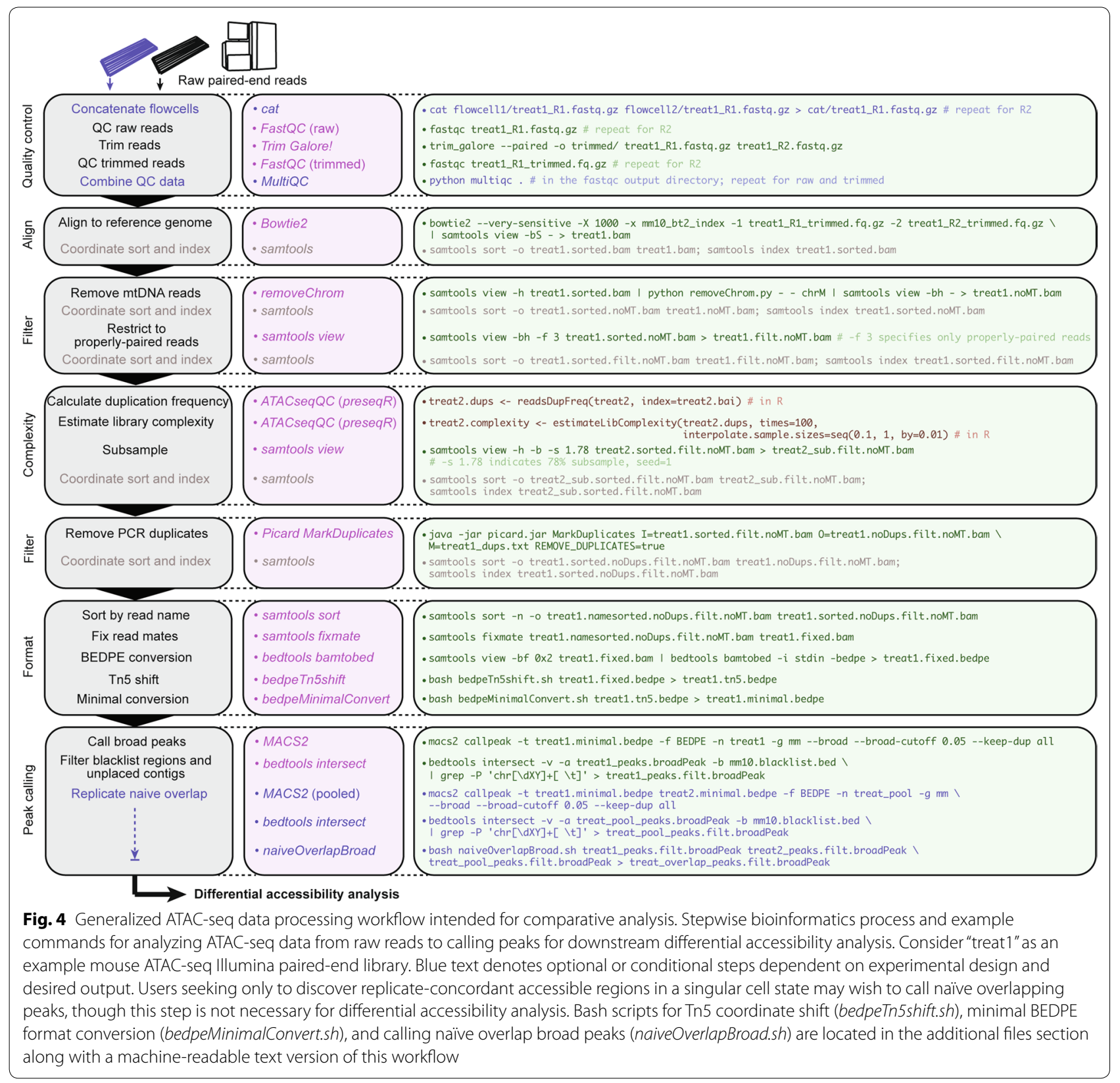

is desired to quantify and compare ATAC signal at different genomic loci which are not typically part of an ATAC-seq or differential ATAC-seq analysis framework. This could involve genomic tiling, i.e., quantifying signal in evenly distributed genomic intervals, or quantifying ATAC at regions defined through other assays, such as ChIP-seq, or $k$-means clustering [20, 43-45]. For certain analyses, it may not be appropriate to implement one of the normalization methods described herein, as determining proper biological or statistical assumptions may not be plausible. Rather, a linear transformation approach is often implemented, such as scaling libraries by read depth. However, this method assumes no differences in global library preparation biases, such as differing ATAC reaction efficiency. A potential result of such biases is affected sequence diversity which manifests in library molecular complexity, or the estimated number of sequenced molecules as determined by duplication rates. If uncorrected, libraries of equivalent read depth may be confounded by complexity in downstream analysis. Instead, libraries can be normalized to the estimated number of sequenced molecules, determined by 
duplication rates as the library molecular complexity. This method mitigates potential biases by considering that the relative distribution of transposase integration (reads at a given feature) is most biologically informative under an equivalent number of transposase reactions (total molecules sequenced, i.e., molecular complexity).

We suggest estimating the complexities of all samples in the compared conditions, and then performing a stochastic subsampling process in order to normalize all samples to equivalent molecular complexity. All analyses presented in this study have undergone this step unless specifically stated otherwise. The R packages preseq $R$ and a wrapper $A T A C s e q Q C$ have implemented functions to estimate complexity by calculating a duplicate frequency matrix then estimating the number of unique molecules sequenced (i.e., molecular complexity) in each library sample $[25,46]$. samtools view can then be used to subsample libraries based on these estimates. In support that the stochastic subsampling process should not greatly affect experimental results, a replicated analysis with two different random subsampling seeds yielded highly similar and overlapping results from peak calling (Additional file 1: Figure S5a) and DA analysis (Additional file 1: Figure S5b).

Among our sorted mouse epithelial cell ATAC-seq data set, control libraries had lower molecular complexities than mutant libraries (Additional file 1: Figure S6a, b), which we corrected by subsampling (Additional file 1: Figure S6c). By performing this complexity-normalization process, we had improved confidence that the observed ATAC differences were biological and not technical in nature. An example of the functional effects of complexity normalization is illustrated through ATAC signal quantification at a set of significant DA promoter regions $(\mathrm{FDR}<0.10)$ defined by approach $I V .35$ promoter regions calculated as significantly decreasing accessibility by this method did not yield statistical significance when quantifying ATAC RPKM in read depth-normalized libraries (Additional file 1: Figure S6d), but the decreasing accessibility patterns become more evident when we compare complexity-normalized libraries $(p=0.0196$, two-tailed paired Wilcoxon test) (Additional file 1: Figure S6e). This analysis highlights the confounding effects of library molecular complexity in comparative ATACseq analysis. Moreover, it supports the use of complexitynormalized libraries for certain quantitative purposes in particular.

\section{Proposed workflow effectively retains ATAC-seq peak calls in an independent data set}

To further assess the effectiveness of our ATAC-seq data analysis workflow, we tested it on one of the original reported data sets generated by Buenrostro et al. [8].
ATAC-seq libraries were generated on three replicates of 50,000 GM12878 human lymphoblastoid cells, and the reported bioinformatic analysis yielded a replicatemerged peak set of 99,885 accessible chromatin regions via $Z I N B A$ [47]. From these data, we are able to assay the ability for our proposed workflow to identify biologically relevant ATAC-seq peaks. Through our workflow, we identified 20,945 genomic regions which MACS2 called a significant broad peak in all three replicates, of which 20,909 (99.8\%) were also retained in the naïve overlap peak set indicating replicate peak region concordance $>50 \%$ (Fig. 5a). To directly compare hg38-aligned naïve overlap peaks called through our workflow with the hg19-aligned Buenrostro et al. ZINBA peak set, we lifted the hg19 coordinates to hg38 with $99.96 \%$ successful mapping rate [48]. We identified extremely strong concordance between the naive overlap peak set and Buenrostro et al. ZINBA peak set, with over $97 \%$ of the naïve overlap peaks intersecting (Fig. $5 \mathrm{~b}$ ). This indicated that nearly all of the naïve overlap peak set regions were also identified with the Buenrostro et al. ZINBA peak set, but there were an additional nearly 80,000 peaks which were not identified in the naïve overlap peak set. When the two peak sets were annotated via HOMER [38], we observed even stronger concordance and conservation between the genes with an identified promoter ATACseq peak in both peak sets. Whereas only roughly $20 \%$ of the genome-wide Buenrostro et al. ZINBA peaks intersected with naïve overlap peaks, approximately $60 \%$ of the genes with an identified promoter ATAC-seq peak were also identified in the naïve overlap peak set (Fig. 5c).

By leveraging a GM12878 microarray gene expression data set from Ernst et al. [49], we were able to next compare the expression of genes which were identified as having a promoter ATAC-seq peak concordantly or uniquely between the two peak sets. Again, we observed strong concordance between the genes measured for expression by microarray which had a promoter ATACseq peak in the two peak sets $(p=0$, hypergeometric enrichment), where 5508 were concordantly identified between both, 2585 were uniquely called in the Buenrostro et al. ZINBA peak set, and 161 were uniquely called in the naive overlap peak set (Fig. $5 \mathrm{~d}$ ). We then compared the expression of the genes in each of these three bins. Genes were equivalently highly expressed that were concordantly identified as exhibiting a promoter ATAC between both peak sets or uniquely in the naive overlap peak set, but the 2585 genes which were uniquely identified as having a promoter ATAC peak in the Buenrostro et al. ZINBA peak set were lowly expressed (Fig. 5e). Furthermore, all genes with a promoter ATAC peak in either of the peak sets were indeed overall more highly expressed than genes which never exhibited a promoter 


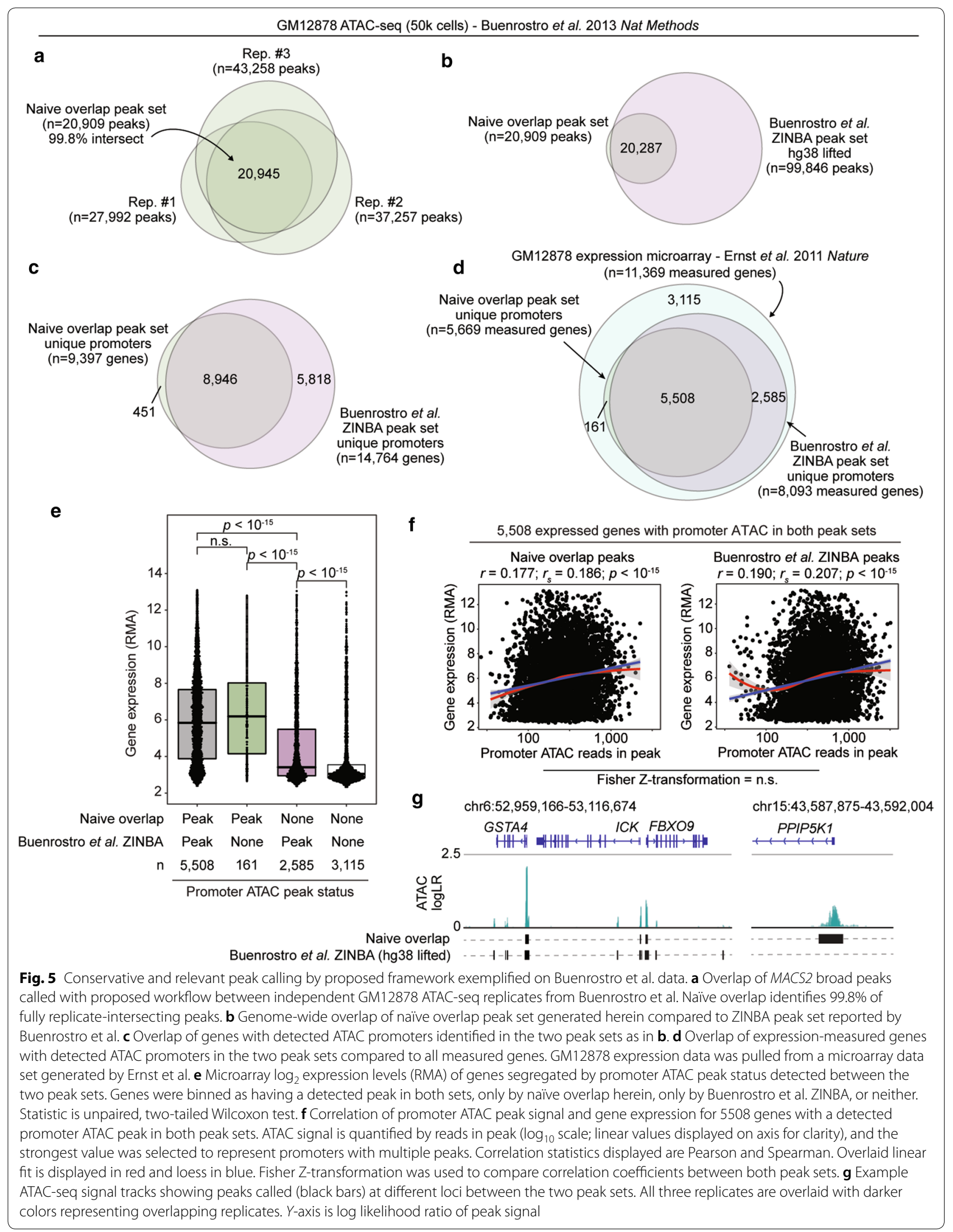


ATAC peak. A further extension of this analysis compared the correlation between promoter ATAC peak signal and respective gene expression for the 5508 commonly identified genes with microarray expression data, as it is widely accepted that higher promoter chromatin accessibility generally corresponds to higher gene expression. Correlations between promoter ATAC peak signal and gene expression were highly significant in both peak sets and analytical approaches and were not significantly different (Fig. 5f). We further observed that the naïve overlap peak set typically identified only conservative ATAC-seq peaks, yet there were also examples of robust ATAC signal which were only called as significant with the naïve overlap peak set, such as near the PPIP5K1 promoter (Fig. 5g). Altogether, these analyses suggest that the workflow proposed herein is able to identify conservative regions of significant ATAC signal which corroborate gene expression observations.

\section{csaw differential accessibility workflow permits testing of multiple normalization methods}

We suggest using csaw as a go-to toolkit for standard downstream differential accessibility analysis. csaw is a flexible R package, originally designed for ChIP-seq analysis, which accepts sorted BAM files for DA quantification via edgeR quasi-likelihood methodology following any one of numerous implemented normalization methods to address many biological scenarios $[28,30]$. Furthermore, csaw can be supplied MACS2 peak coordinates for DA analysis or alternatively perform de novo ATAC enrichment detection with sliding windows and proper type I error control. These features make csaw an attractive tool for comprehensive DA analysis. For DA analysis, we have graphically represented a typical csaw workflow in $\mathrm{R}$ (Fig. 6), which is also available as a machine-readable text version (Additional file 6). This workflow outlines a continuation of Fig. 4 into complete DA analysis which aids users to compute DA approaches III, $I V, V$, and $V I$ for output evaluation. The sensitivity of the proposed workflows in distinguishing signal from noise is further evident in DA analysis of two independent groups of negative controls from the Schep et al. yeast ATAC-seq data set (see Additional file 1: Figure S2).

The final steps of this graphic detail generation of an MA plot through ggplot2 [50] to assess normalization outcomes. The distribution of an MA plot from DA analysis provides insight into trends or potential biases within the data, which users should critically consider. An upward or downward shift in the MA distribution could either indicate a global effect or substantial technical bias, and certain normalization methods will maintain or eliminate these features in the data. If the distribution further does not appear symmetrical along the horizontal axis, then a trended bias may be present which can be corrected with conservative normalization approaches like quantile or loess. Ultimately, the researcher must consider prior biological knowledge and the experimental design to determine if data trends should be accepted as biological or eliminated as technical.

\section{Discussion}

This study has revealed that differential accessibility analysis of ATAC-seq data can be sensitive to underlying biases within the data, as might be expected. The design of the primary analyzed data set involved disrupting a chromatin remodeler subunit, which probably affects genome-scale chromatin structure, and comparing chromatin accessibility to that of control cells. Analytical interpretation is further confounded by in vivo heterogeneity in sorted cell populations. The consequence is that common tools and approaches for performing DA analysis give vastly different results that are difficult to interpret at first glance. MA plots of DA results displayed global ATAC distribution biases that were only thoroughly eliminated through certain normalization methods, like loess-based count adjustments. By comparing multiple DA analysis outputs, common patterns emerged that permitted high likelihood conclusions, such as gene promoters increasing in accessibility following chromatin remodeler subunit disruption. Application of precision-recall analysis to predict RNA-seq gene expression changes with DA data supported biological relevance of certain DA methods, since gene expression and promoter chromatin accessibility are known to correlate. This also further emphasized that pairing ATAC-seq with RNAseq can be a useful approach to interpreting chromatin accessibility observations. FDR thresholding analyses suggest that optimizing the DA significance threshold can also improve results and interpretation. Overall, these results indicate that naively relying on one single DA analysis approach may lead to false conclusions, particularly so without assessing for presence of biases within the data. In the case of modifying or disrupting chromatin regulators, these biases may be commonplace and should be critically considered before further interpretation of data. Furthermore, certain methods are more conservative than others and can be initially selected to improve result confidence without the need to perform rigorous method comparisons.

The issue of intrinsic biases within ATAC-seq data for experimental designs where global changes may be expected are difficult to interpret. In the case of the experimental data presented here, limited prior knowledge is available whether or not disruption of this specific chromatin remodeler subunit should affect widespread chromatin structure, but it is not improbable. Thus, we 


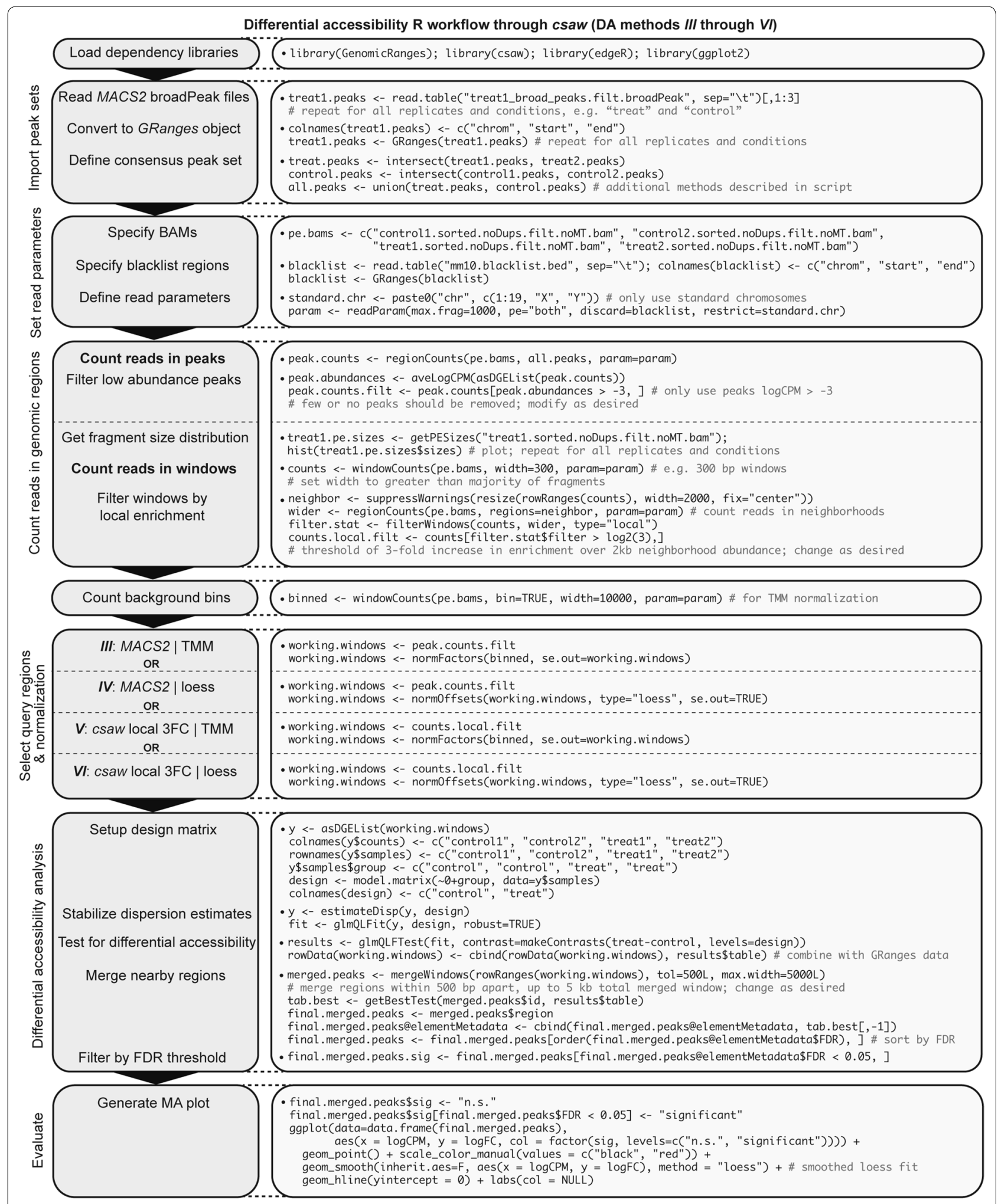

Fig. 6 csaw workflow for multiple differential accessibility analyses in R. Graphical representation of proposed csaw workflow in R for calculating differential accessibility. Consider an experimental design with $n=2$ biological replicates from two conditions: "treat" and "control" 
were not able to determine if the inherent biases within the data are biological or technical, and we opted to remove them as technical. However, this decision could result in significant type II errors interpreted as technical in origin, if in fact they are truly related to the biology. Multiple downstream comparative analyses supported the loess normalization method as conservative and biologically relevant to our data set, so it was chosen as the normalization method with which to proceed forward for our published downstream analysis [17]. Though, significant information may have been lost by eliminating the global, trended biases that were observed within the data.

The observation that comparing ATAC-seq samples between two conditions may be subject to substantial experimental biases was actually accounted by Schep et al. in the yeast osmotic stress differential ATAC-seq report [18]. The authors specifically noted that, 'variation in the degree of enrichment of fragments with open chromatin regions can affect differential accessibility measurements between ATAC-seq samples. As such, they followed count quantile normalization with a lowess curve fit transformation to eliminate trended biases within the data. This account further supports that differential ATAC-seq analyses are sensitive to experimental, technical biases, such as ATAC reaction efficiency, as well as the rationale behind use of the loess normalization method to elicit a highly conservative DA result. Still, this normalization method is not widely used for ATAC-seq DA analysis. It is also important to note that similar observations have been reported for ChIP-seq analysis, where non-linear loess normalization methods were proposed and developed to eliminate systematic errors between libraries [51]. However, the rationale behind a loess fit assumes that the data should be symmetrical without a global change observed, so users should be aware that implementing this technique may hide any true global alterations present between the two conditions.

Biases inherent to quantitative genomic techniques based on chromatin feature signal enrichment have been observed and considered previously [52]. ATAC-seq fundamentally relies on an enzymatic reaction for library construction, which is likely to be affected by amount of enzyme, number of nuclei, and chromatin compaction and structure. ATAC-seq was recently reported to exhibit a sequence-specific bias distinct from DNase I libraries [53]. MNase digestion has previously been shown to be highly sensitive to enzymatic activity and also displays sequence specificity bias $[54,55]$. In ChIP-seq libraries, potential bias in factor binding measurements is thought to be derived from local transcriptional activity and chromatin structural properties [56]. Our current investigation has shown that quantitative comparative analysis of
ATAC libraries is confounded by technical bias. When alternative methods to detect chromatin accessibility changes are unavailable (e.g., due to low cell numbers or input retrieval), users should empirically determine the most appropriate normalization methods and employ orthogonal assays, such as gene expression, for comparisons with ATAC-seq data.

Calculating linear library normalization factors is a standard approach, but sensitive to biases. Here, we have shown that MA plots are a simple, qualitative approach to identifying systematic biases in experimental ATAC-seq libraries, as others have shown with ChIP-seq data [57]. Calculating the fraction of reads in peaks (FRiP) score, as described by ENCODE [41] and streamlined by DiffBind, is a simple method to evaluating ATAC efficiency between libraries to determine whether or not a systematic bias may be present. In the case of substantially differing ATAC efficiencies, linear normalization factors can be derived from only reads in peaks, as in DiffBind, or by applying TMM to only high abundance regions, as is suggested by the authors of csaw. As we have discussed, csaw also has a non-linear loess-based count normalization which can be easily implemented to assess its effects on DA calculation after the above considerations.

Before DA analysis, the most significant addition to our proposed standard ATAC-seq data analysis workflow is normalization of library complexity by random subsampling. In the case that ATAC reaction efficiencies are different between libraries, it is advised to investigate the library molecular complexity as a technical source for this error arising during sequencing. If less input material is retrieved from transposition for certain samples, and more PCR amplification cycles are required as a result [58], then bias is introduced into the amplified fragments dependent on GC content, fragment length, and oligonucleotide complexity [59]. If libraries are complexity-normalized within an experimental design to the same estimated number of unique molecules, then direct quantitative comparison of unique fragments between conditions is more informative, e.g., integer feature read counts at loci identified in other assays. Like the systematic biases present in DA analysis, however, library complexity normalization is currently also a flawed concept. In the case that a drastic global decrease in chromatin accessibility is truly biological, then less transposed DNA fragment retrieval is expected, and these libraries might exhibit lower complexity. In this scenario, complexity normalization may not be desired as it would confound the true chromatin biology. However, without independent knowledge, this decision is not easily made. Notably, others have approached similar problems in ChIP-seq through addition of exogenous chromatin from a distinct species as reference for IP efficiency and sequencing bias, 
referred to as "spike-in" controls [60, 61]. More recently, this technique has been extended to incorporating a fraction of spike-in live cells prior to lysis in the ATAC-seq protocol [62], and the effects of spike-in normalization of ATAC-seq data could help establish technical or biological basis for global accessibility patterns, in principle.

The presented ATAC-seq workflow and the suggested DA toolkit are not absolute and should be improved as analytical methods continue to emerge. For example, one newly developed method implementing a hidden Markov model (HMM) showed better performance for differential ChIP-seq analysis than csaw or other sliding window approaches, which suffer in identifying narrow changes within large genomic domains [63]. At least one HMM tool has been developed specifically for calling nucleosomefree regions within ATAC-seq data, and its implementation could be extended to differential analysis [64]. While our framework was able to identify both broad and narrow regions of strong ATAC signal in the Buenrostro et al. GM12878 data set, the peak calling thresholds may be too strict to identify truly nucleosome-depleted regions displaying weak signal. Methods also currently exist for correcting sequence-specific biases resulting from various chromatin digestion and enrichment techniques, and the extent of analytical affect from this correction should be evaluated $[65,66]$. Currently, ATAC-seq normalization and DA approaches should be carefully considered to appropriately reduce the inherent biases within each analysis.

\section{Conclusions}

We present data indicating that ATAC-seq is sensitive to bias when comparing chromatin accessibility across multiple conditions. We compared several commonly used, published methods for calculating differential accessibility to our previously reported in vivo ATACseq data set as well as a yeast ATAC-seq time series data set, and we observe conflicting results dependent upon the normalization method used. We provide intuitive, standardized bioinformatics methodology for analyzing ATAC-seq data by non-computational scientists. Our validated workflow also includes a critical, complexitynormalization step. Altogether, we argue that researchers should properly normalize ATAC-seq data before calculating differential accessibility.

\section{Methods}

\section{Analyzed data sets}

Sequencing data used for analyses presented in this manuscript were downloaded from GEO accessions GSE121198, GSE66386, GSE47753, and GSE26312. Yeast genes with distinct expression response patterns following $0.6 \mathrm{M} \mathrm{NaCl}$ exposure were defined and extracted from Supp. Table 4 reported by Ni et al. [40]

\section{ATAC-seq and differential accessibility analysis}

See Figs. 4 and 6, and Additional file 1: Method for complete workflow details and description. Mouse libraries were aligned to $\mathrm{mm} 10$ genome assembly, and yeast libraries were aligned to sacCer3 genome assembly. ATAC-seq peaks were not filtered for blacklisted regions in yeast, as they are not defined in this organism. Presented DA analyses were computed through the use of $\mathrm{R}$ packages DiffBind, DESeq2, csaw, edgeR, voom, and limma as described in the "Results" section [28-33]. Workflows for all tools are described in detail in Additional file 1: Methods. The BAM files supplied to DA tools correspond to the coordinate sorted/indexed, duplicate removed, complexity-normalized, properly paired restricted, non-mitochondrial, paired-end BAM files generated as described in Fig. 4.

\section{RNA-seq analysis}

Differential gene expression results from previously reported RNA-seq data of $L t f C r e^{0 /+} ;(G t) R 26^{P i k 3 c a^{*} H 1047 R}$; Arid $1 a^{f l f l}$ vs. control sorted mouse endometrial epithelial cells were extracted from GEO accession GSE129784. 3481 significant DE genes were selected by FDR $<0.05$ filtering. 24,097 total expressed genes were used as gene universe for enrichment analyses.

\section{GM12878 gene expression microarray analysis}

Raw data were downloaded from GEO for both GM12878 replicates generated by Affymetrix HT Human Genome U133A Array. CEL files were read into $R$ through the affy package and normalized via the Robust Multi-Array Average (RMA) expression measure. RMA values are reported as $\log _{2}$ scale. The mean RMA value of both replicates was used for analyses in this manuscript. The 22,277 measured probes were collapsed to 11,369 genes with a unique Ensembl and symbol identifier [67]. Expression comparisons of gene groups binned by ATAC peak status was achieved by unpaired, two-tailed Wilcoxon test.

\section{Bioinformatics and statistics}

Mouse and human ATAC-seq peak coordinates were annotated by HOMER [38] with a modification to cispromoter classification as within $3000 \mathrm{bp}$ of a canonical gene TSS. Yeast genomic regions were annotated by TxDb.Scerevisiae.UCSC.sacCer3.sgdGene $\mathrm{R}$ package using the genes() and promoters() functions with default settings (yeast promoters are defined as -2000 to $+200 \mathrm{bp}$ around TSS) [68]. Unweighted precisionrecall curves were generated by the $P R R O C \mathrm{R}$ package using the pr.curve() function [69]. For PR curve predictive analysis, the strongest (lowest) promoter DA FDR value was selected for each expressed gene, for each 
approach, and this value was used to predict boolean DE gene status segregated by RNA-seq DGE DESeq2 FDR $<0.05$ threshold. Read counts in ATAC-seq peaks were calculated by $H O M E R$ for correlation and box dot plot quantification. MACS2 was used to generate ATAC-seq signal tracks for display in $I G V[27,70]$. MSigDB Hallmark pathway enrichment was reported as observed/expected ratios derived from expressed gene sets compared to the respective expressed gene universe [71]. Pathway hierarchical clustering by Euclidean distance and heatmap were generated by ComplexHeatmap [72]. biomaRt was used for all gene nomenclature and mouse-human ortholog conversions [73]. The cumulative hypergeometric distribution was calculated in R for enrichment tests. ggplot 2 was used for certain plotting applications throughout this manuscript [50].

\section{Supplementary information}

Supplementary information accompanies this paper at https://doi. org/10.1186/s13072-020-00342-y.

Additional file 1. Supplementary Information (Methods and Figures).

Additional file 2. ATACseq_workflow.txt-Example machine-readable Fig. 4 workflow including stepwise unix and R commands for ATAC-seq data processing.

Additional file 3. bedpeTn5shift.sh—Bash script for shifting coordinates in standard 10-column format BEDPE files to compensate for Tn5 adapter insertion as described in Buenrostro et al. See Fig. 4 for usage.

Additional file 4. bedpeMinimalConvert.sh-Bash script for converting standard 10-column format BEDPE to the "minimal" format defined by MACS2. See Fig. 4 for usage.

Additional file 5. naiveOverlapBroad.sh—Bash script for calculating naïve overlap broad peak set from 2 individual replicate peak sets and a pooled replicate peak set. Can be modified for to accept more replicates as desired. See Fig. 4 for usage.

Additional file 6. csaw_workflow.R_Example R workflow for differential accessibility analysis with csaw as graphically displayed in Fig. 6. Describes process for both TMM and loess normalizations and either supplying MACS2 peak sets as query regions or identifying de novo locally enriched windows.

\section{Abbreviations}

ATAC: Assay for Transposase-Accessible Chromatin; DA: Differential accessibility; differentially accessible; DE: Differential expression; differentially expressed; EMT: Epithelial-mesenchymal transition; FRiP: Fraction of reads in peaks; GLM: Generalized linear model; HMM: Hidden Markov model; loess: Locally estimated scatterplot smoothing; $\log _{2}$ CPM: Log $_{2}$ counts per million; logCPM; $\log _{2} \mathrm{FC}$ : Log 2 fold change; logFC; lowess: Locally weighted scatterplot smoothing; MNase: Micrococcal nuclease; PR: Precision-recall; RPK: Reads per kilobase; RPKM: Reads per kilobase per million mapped reads; TMM: Trimmed mean of $M$ values.

\section{Acknowledgements}

We thank Drs. Ben Johnson and Tim Triche, Jr. for helpful discussions.

\section{Authors' contributions}

$J J R, M R W$, and RLC conceptualized and initiated the study. JJR devised the methodology and performed all formal data curation and analysis present in this manuscript. MRW generated the primary analyzed sorted mouse ATACseq libraries which were previously reported. JJR wrote the manuscript with revisions from MRW and RLC. RLC secured funding and supervised the study. All authors read and approved the final manuscript.

\section{Funding}

R.L.C. was supported by an Innovative Translational Grant from the Mary Kay Foundation (026-16), Liz Tilberis Early Career Award from the Ovarian Cancer Research Fund Alliance (OCRFA) (457446), and the NIH National Institute for Child Health and Human Development (HD099383-01).

\section{Availability of data and materials}

All analyzed data sets are publicly available at GEO accessions GSE121198, GSE66386, GSE47753, and GSE26312. Yeast genes with distinct expression response patterns following $0.6 \mathrm{M} \mathrm{NaCl}$ exposure were defined and extracted from Supp. Table 4 reported by Ni et al. [73]. All workflow and custom function scripts are available in the "Additional files" section as well as a GitHub repository (https://github.com/reskejak/ATAC-seq). A detailed description of the workflow commands in Figs. 4 and 6 as well as all different DA analysis methods are available in Additional file 1: Methods.

\section{Ethics approval and consent to participate}

Not applicable.

\section{Consent for publication}

Not applicable.

\section{Competing interests}

The authors declare no competing interests.

\section{Author details}

${ }^{1}$ Department of Obstetrics, Gynecology and Reproductive Biology, College of Human Medicine, Michigan State University, Grand Rapids, MI 49503, USA. ${ }^{2}$ Center for Epigenetics, Van Andel Research Institute, Grand Rapids, MI 49503, USA.

Received: 31 December 2019 Accepted: 11 April 2020

Published online: 22 April 2020

\section{References}

1. Wang Z, Gerstein M, Snyder M. RNA-Seq: a revolutionary tool for transcriptomics. Nat Rev Genet. 2009:10:57-63.

2. Laird PW. Principles and challenges of genomewide DNA methylation analysis. Nat Rev Genet. 2010;11:191-203.

3. Park PJ. ChIP-seq: advantages and challenges of a maturing technology. Nat Rev Genet. 2009:10:669-80.

4. O'Geen H, Echipare L, Farnham PJ. Using ChIP-seq technology to generate high-resolution profiles of histone modifications. Methods Mol Biol. 2011;791:265-86

5. Lieberman-Aiden $\mathrm{E}$, et al. Comprehensive mapping of long-range interactions reveals folding principles of the human genome. Science. 2009:326:289-93.

6. Henikoff JG, Belsky JA, Krassovsky K, MacAlpine DM, Henikoff S. Epigenome characterization at single base-pair resolution. Proc Natl Acad Sci USA. 2011;108:18318-23.

7. Boyle AP, et al. High-resolution mapping and characterization of open chromatin across the genome. Cell. 2008;132:311-22.

8. Buenrostro JD, Giresi PG, Zaba LC, Chang HY, Greenleaf WJ. Transposition of native chromatin for fast and sensitive epigenomic profiling of open chromatin, DNA-binding proteins and nucleosome position. Nat Methods. 2013;10:1213-8.

9. Klemm SL, Shipony Z, Greenleaf WJ. Chromatin accessibility and the regulatory epigenome. Nat Rev Genet. 2019;20:207-20.

10. Tsompana M, Buck MJ. Chromatin accessibility: a window into the genome. Epigenet Chromatin. 2014;7:33.

11. Corces MR, et al. An improved ATAC-seq protocol reduces background and enables interrogation of frozen tissues. Nat Methods. 2017;14:959-62.

12. Jen $\mathrm{HI}$, et al. Transcriptomic and epigenetic regulation of hair cell regeneration in the mouse utricle and its potentiation by Atoh1. Elife. 2019;8:e44328. 
13. Frerichs A, Engelhorn J, Altmuller J, Gutierrez-Marcos J, Werr W. Specific chromatin changes mark lateral organ founder cells in the Arabidopsis inflorescence meristem. J Exp Bot. 2019;70:3867-79.

14. Hilliard S, et al. Defining the dynamic chromatin landscape of mouse nephron progenitors. Biol Open. 2019;8:42754

15. Jia G, et al. Single cell RNA-seq and ATAC-seq analysis of cardiac progenitor cell transition states and lineage settlement. Nat Commun. 2018;9:4877.

16. Haines RR, et al. The histone demethylase LSD1 regulates B cell proliferation and plasmablast differentiation. J Immunol. 2018;201:2799-811.

17. Wilson MR, et al. ARID1A and PI3-kinase pathway mutations in the endometrium drive epithelial transdifferentiation and collective invasion. Nat Commun. 2019;10:3554.

18. Schep AN, et al. Structured nucleosome fingerprints enable high-resolution mapping of chromatin architecture within regulatory regions. Genome Res. 2015;25:1757-70.

19. Liu C, et al. An ATAC-seq atlas of chromatin accessibility in mouse tissues. Sci Data. 2019;6:65

20. Corces MR, et al. The chromatin accessibility landscape of primary human cancers. Science. 2018;362:1898.

21. Yan F, Powell DR, Curtis DJ, Wong NC. From reads to insight: a hitchhiker's guide to ATAC-seq data analysis. Genome Biol. 2020;21:22.

22. Gusmao EG, Allhoff M, Zenke M, Costa IG. Analysis of computational footprinting methods for DNase sequencing experiments. Nat Methods. 2016;13:303-9.

23. Divate M, Cheung E. GUAVA: a Graphical User Interface for the Analysis and Visualization of ATAC-seq Data. Front Genet. 2018;9:250.

24. Ahmed Z, Ucar D. I-ATAC: interactive pipeline for the management and preprocessing of ATAC-seq samples. PeerJ. 2017;5:e4040.

25. Ou J, et al. ATACseqQC: a Bioconductor package for post-alignment quality assessment of ATAC-seq data. BMC Genomics. 2018;19:169.

26. Pranzatelli TJF, Michael DG, Chiorini JA. ATAC2GRN: optimized ATAC-seq and DNase1-seq pipelines for rapid and accurate genome regulatory network inference. BMC Genomics. 2018;19:563.

27. Zhang Y, et al. Model-based analysis of ChIP-Seq (MACS). Genome Biol. 2008:9:R137.

28. Lun AT, Smyth GK. csaw: a Bioconductor package for differential binding analysis of ChIP-seq data using sliding windows. Nucleic Acids Res. 2016;44:e45.

29. Stark R, Brown G. DiffBind: differential binding analysis of ChIP-seq peak data. R package version. 2011;100:3-4.

30. Robinson MD, McCarthy DJ, Smyth GK. edgeR: a Bioconductor package for differential expression analysis of digital gene expression data. Bioinformatics. 2010;26:139-40.

31. Love MI, Huber W, Anders S. Moderated estimation of fold change and dispersion for RNA-seq data with DESeq2. Genome Biol. 2014;15:550.

32. Law CW, Chen Y, Shi W, Smyth GK. voom: precision weights unlock linear model analysis tools for RNA-seq read counts. Genome Biol. 2014;15:R29.

33. Ritchie ME, et al. limma powers differential expression analyses for RNAsequencing and microarray studies. Nucleic Acids Res. 2015;43:e47.

34. Robinson MD, Oshlack A. A scaling normalization method for differential expression analysis of RNA-seq data. Genome Biol. 2010;11:R25.

35. Bolstad BM, Irizarry RA, Astrand M, Speed TP. A comparison of normalization methods for high density oligonucleotide array data based on variance and bias. Bioinformatics. 2003;19:185-93.

36. Wilson MR, Holladay J, Chandler RL. A mouse model of endometriosis mimicking the natural spread of invasive endometrium. Hum Reprod. 2020:35:58-69.

37. Dudoit SY, Callow MJ, Speed TP. Statistical methods for identifying differentially expressed genes in replicated cdna microarray experiments. Statist Sinica. 2002;12:111-39.

38. Heinz $\mathrm{S}$, et al. Simple combinations of lineage-determining transcription factors prime cis-regulatory elements required for macrophage and B cell identities. Mol Cell. 2010;38:576-89.

39. Li B, Carey M, Workman JL. The role of chromatin during transcription. Cell. 2007:128:707-19.

40. Ni L, et al. Dynamic and complex transcription factor binding during an inducible response in yeast. Genes Dev. 2009;23:1351-63.

41. Landt SG, et al. ChIP-seq guidelines and practices of the ENCODE and modENCODE consortia. Genome Res. 2012;22:1813-31.
42. Amemiya HM, Kundaje A, Boyle AP. The ENCODE blacklist: identification of problematic regions of the genome. Sci Rep. 2019;9:9354.

43. Kelso TWR, et al. Chromatin accessibility underlies synthetic lethality of SWI/ SNF subunits in ARID1A-mutant cancers. Elife. 2017;6:e30506.

44. Hosoya T, et al. Global dynamics of stage-specific transcription factor binding during thymocyte development. Sci Rep. 2018;8:5605.

45. Toenhake CG, et al. chromatin accessibility-based characterization of the gene regulatory network underlying plasmodium falciparum blood-stage development. Cell Host Microbe. 2018;23:557-69.

46. Daley T, Smith AD. Predicting the molecular complexity of sequencing libraries. Nat Methods. 2013;10:325-7.

47. Rashid NU, Giresi PG, Ibrahim JG, Sun W, Lieb JD. ZINBA integrates local covariates with DNA-seq data to identify broad and narrow regions of enrichment, even within amplified genomic regions. Genome Biol. 2011;12:R67.

48. Kent WJ, et al. The human genome browser at UCSC. Genome Res. 2002;12:996-1006

49. Ernst J, et al. Mapping and analysis of chromatin state dynamics in nine human cell types. Nature. 2011;473:43-9.

50. Wickham H. ggplot2: elegant graphics for data analysis, 212. New York: Springer-Verlag; 2016.

51. Taslim C, et al. Comparative study on ChIP-seq data: normalization and binding pattern characterization. Bioinformatics. 2009;25:2334-40.

52. Meyer CA, Liu XS. Identifying and mitigating bias in next-generation sequencing methods for chromatin biology. Nat Rev Genet. 2014;15:709-21.

53. Karabacak Calviello A, Hirsekorn A, Wurmus R, Yusuf D, Ohler U. Reproducible inference of transcription factor footprints in ATAC-seq and DNase-seq datasets using protocol-specific bias modeling. Genome Biol. 2019;20:42.

54. Mieczkowski J, et al. MNase titration reveals differences between nucleosome occupancy and chromatin accessibility. Nat Commun. 2016;7:11485.

55. Dingwall C, Lomonossoff GP, Laskey RA. High sequence specificity of micrococcal nuclease. Nucleic Acids Res. 1981;9:2659-73.

56. Park D, Lee Y, Bhupindersingh G, lyer VR. Widespread misinterpretable ChIPseq bias in yeast. PLoS ONE. 2013;8:e83506.

57. Shao Z, Zhang Y, Yuan GC, Orkin SH, Waxman DJ. MAnorm: a robust model for quantitative comparison of ChIP-Seq data sets. Genome Biol. 2012;13:R16.

58. Buenrostro JD, Wu B, Chang HY, Greenleaf WJ. ATAC-seq: a method for assaying chromatin accessibility genome-wide. Curr Protoc Mol Biol. 2015;109:1-9.

59. Aird $D$, et al. Analyzing and minimizing PCR amplification bias in Illumina sequencing libraries. Genome Biol. 2011;12:R18.

60. Orlando DA, et al. Quantitative ChIP-Seq normalization reveals global modulation of the epigenome. Cell Rep. 2014;9:1163-70.

61. Chen $\mathrm{K}$, et al. The overlooked fact: fundamental need for spike-in control for virtually all genome-wide analyses. Mol Cell Biol. 2015;36:662-7.

62. Stewart-Morgan KR, Reveron-Gomez N, Groth A. Transcription restart establishes chromatin accessibility after DNA replication. Mol Cell. 2019;75:408-14

63. Allhoff M, Sere K, Zenke M. Differential peak calling of ChIP-seq signals with replicates with THOR. Nucleic Acids Res. 2016;44:e153.

64. Tarbell ED, Liu T. HMMRATAC: a hidden markov ModeleR for ATAC-seq. Nucleic Acids Res. 2019;47:e91.

65. Wang JR, Quach B, Furey TS. Correcting nucleotide-specific biases in highthroughput sequencing data. BMC Bioinf. 2017;18:357.

66. Martins AL, Walavalkar NM, Anderson WD, Zang C, Guertin MJ. Universal correction of enzymatic sequence bias reveals molecular signatures of protein/ DNA interactions. Nucleic Acids Res. 2018;46:e9.

67. Zerbino DR, et al. Ensembl 2018. Nucleic Acids Res. 2018;46:D754-61.

68. Carlson M. Bioconductor Package Maintainer. TxDb.Scerevisiae.UCSC.sacCer3.sgdGene: Annotation package for TxDb object(s). R package version 3.2.2. (2015).

69. Grau J, Grosse I, Keilwagen J. PRROC: computing and visualizing precisionrecall and receiver operating characteristic curves in R. Bioinformatics. 2015:31:2595-7.

70. Robinson JT, et al. Integrative genomics viewer. Nat Biotechnol. 2011;29:24-6.

71. Liberzon A, et al. The molecular signatures database (MSigDB) hallmark gene set collection. Cell Syst. 2015;1:417-25. 
72. Gu Z, Eils R, Schlesner M. Complex heatmaps reveal patterns and correlations in multidimensional genomic data. Bioinformatics. 2016;32:2847-9.

73. Smedley D, et al. BioMart-biological queries made easy. BMC Genomics. 2009;10:22.

\section{Publisher's Note}

Springer Nature remains neutral with regard to jurisdictional claims in published maps and institutional affiliations.
Ready to submit your research? Choose BMC and benefit from:

- fast, convenient online submission

- thorough peer review by experienced researchers in your field

- rapid publication on acceptance

- support for research data, including large and complex data types

- gold Open Access which fosters wider collaboration and increased citations

- maximum visibility for your research: over 100M website views per year

At BMC, research is always in progress.

Learn more biomedcentral.com/submissions 